\title{
Erratum to: Treatment of Provoked Vulvodynia in a Swedish cohort using desensitization exercises and cognitive behavioral therapy
}

\author{
Suzanne Lindström ${ }^{1 *}$ and Linda J. Kvist ${ }^{2,3}$
}

After publication of the original article [1] it was brought to our attention that tables two and three (here, Tables 1 and 2) contained incorrect data in question 7 and 11. The correct tables are included below. Changes in the tables do not affect the conclusions drawn in the study.

\begin{abstract}
Author details
'Sexology Department, Najaden Midwifery Clinic, Drottninggatan 7 , Helsingborg 252 21, Sweden. ${ }^{2}$ Department of Obstetrics and Gynecology, Helsingborgs Hospital, Helsingborg 25187, Sweden. ${ }^{3}$ Department of Health Sciences, Faculty of Medicine, Lund University, Lund 22100, Sweden.
\end{abstract}

Received: 11 December 2015 Accepted: 11 December 2015

Published online: 23 December 2015

\section{Reference}

1. Lindström S, Kvist $L$. Treatment of Provoked Vulvodynia in a Swedish cohort using desensitization exercises and cognitive behavioral therapy. BMC Womens Health. 2015;15:108.

\footnotetext{
* Correspondence: suzanne.lindstrom@telia.com

${ }^{1}$ Sexology Department, Najaden Midwifery Clinic, Drottninggatan 7, Helsingborg 252 21, Sweden

Submit your next manuscript to BioMed Central and we will help you at every step:

- We accept pre-submission inquiries

- Our selector tool helps you to find the most relevant journal

- We provide round the clock customer support

- Convenient online submission

- Thorough peer review

- Inclusion in PubMed and all major indexing services

- Maximum visibility for your research

Submit your manuscript at

www.biomedcentral.com/submit 
Table 1 Scores for all questions on the MFSQ (median and range) and comparison of individual question scores before and directly after completed treatment using Wilcoxon signed rank test (paired)

\begin{tabular}{|c|c|c|c|}
\hline \multirow[t]{2}{*}{ Question } & \multirow{2}{*}{$\begin{array}{l}\text { MFSQ (before treatment) } \\
\text { Median (range) }\end{array}$} & \multicolumn{2}{|l|}{ MFSQ (directly after treatment) } \\
\hline & & Median (range) & $p$-value \\
\hline $\begin{array}{l}\text { 1. Are you satisfied with the extent of your sexual life at the present } \\
\text { moment? ( } 1 \text { = very dissatisfied, } 7=\text { very satisfied })\end{array}$ & $\begin{array}{l}2.0(1-6) \\
n=60\end{array}$ & $\begin{array}{l}5.0(1-7) \\
n=59\end{array}$ & $<0.01 *(z-6.50)$ \\
\hline $\begin{array}{l}\text { 2. Approximately how many times, during the last month, have you thought } \\
\text { about sex or had sexual fantasies? ( } 1=\text { never, } 7=>10 \text { times per day) }\end{array}$ & $\begin{array}{l}3.0(1-6) \\
n=60\end{array}$ & $\begin{array}{l}4.0(1-7) \\
n=59\end{array}$ & $<0.01^{*}(z-5.10)$ \\
\hline $\begin{array}{l}\text { 3. Does sexual activity give you pleasure? }(1=\text { no pleasure at all, } \\
7=\text { a lot of pleasure) }\end{array}$ & $\begin{array}{l}4.0(1-7) \\
n=53\end{array}$ & $\begin{array}{l}6.0(13-7) \\
n=53\end{array}$ & $<0.01^{*}(z-5.10)$ \\
\hline $\begin{array}{l}\text { 4. How often do you feel sexually excited or stimulated during sexual activity? } \\
\text { (Example: increased pulse, increased vaginal lubrication, skin redness, quicker } \\
\text { breathing, increased sensitivity in the skin and erogenous areas). } \\
(1=\text { never, } 7=\text { every time) }\end{array}$ & $\begin{array}{l}4.0(1-7) \\
n=53\end{array}$ & $\begin{array}{l}6.0(2-7) \\
n=54\end{array}$ & $<0.01^{*}(z-4.51)$ \\
\hline $\begin{array}{l}\text { 5. How often do you achieve orgasm during sexual activity? (For example } \\
\text { increased pulse, increased vaginal lubrication, skin redness, quicker } \\
\text { breathing, increased sensitivity in the skin and erogenous areas). } \\
\text { ( } 1=\text { never, } 7=\text { every time) }\end{array}$ & $\begin{array}{l}4.0(1-7) \\
n=53\end{array}$ & $\begin{array}{l}6.0(1-7) \\
n=54\end{array}$ & $<0.01^{*}(z-3.85)$ \\
\hline $\begin{array}{l}\text { 6. How often is vaginal dryness a problem during sexual activity? } \\
(1=\text { every time, } 7=\text { never })\end{array}$ & $\begin{array}{l}4.0(1-7) \\
n=54\end{array}$ & $\begin{array}{l}6.0(2-7) \\
n=54\end{array}$ & $<0.01^{*}(z-3.60)$ \\
\hline 7. How often is sexual intercourse painful? ( $1=$ every time, $7=$ never) & $\begin{array}{l}1.0(1-7) \\
n=53\end{array}$ & $\begin{array}{l}6.0(2-7) \\
n=53\end{array}$ & $<0.01^{*}(z-6.01)$ \\
\hline $\begin{array}{l}\text { 8. Are you satisfied with your partner as a lover? ( } 1 \text { = very dissatisfied, } \\
7 \text { = very satisfied) }\end{array}$ & $\begin{array}{l}7.0(2-7) \\
n=55\end{array}$ & $\begin{array}{l}6.0(1-7) \\
n=54\end{array}$ & $0.75(z-0.32)$ \\
\hline $\begin{array}{l}\text { 9. Are you satisfied with your partner as a fellow human and friend? } \\
(1=\text { very dissatisfied, } 7 \text { = very satisfied })\end{array}$ & $\begin{array}{l}7.0(2-7) \\
n=54\end{array}$ & $\begin{array}{l}7.0(2-7) \\
n=54\end{array}$ & $0.06(z-1.90)$ \\
\hline $\begin{array}{l}\text { 10. How often have you had sexual intercourse during the last month? } \\
(1=\text { never, } 6=>8 \text { times) }\end{array}$ & $\begin{array}{l}2.0(1-6) \\
n=60\end{array}$ & $\begin{array}{l}3.0(1-6) \\
n=59\end{array}$ & $<0.01^{*}(z-4.51)$ \\
\hline $\begin{array}{l}\text { 11. How often have you satisfied yourself, alone, during the last month? } \\
(1=\text { never, } 6=>8 \text { times) }\end{array}$ & $\begin{array}{l}1.5(1-6) \\
n=60\end{array}$ & $\begin{array}{l}4.0(1-6) \\
n=59\end{array}$ & $<0.01^{*}(z-5.90)$ \\
\hline $\begin{array}{l}\text { 12. How often do you avoid intercourse in order to avoid pain? } \\
\text { ( } 1=\text { always, } 5=\text { never })\end{array}$ & $\begin{array}{l}2.0(1-5) \\
n=58\end{array}$ & $\begin{array}{l}4.0(12-5) \\
n=52\end{array}$ & $<0.01^{*}(z-5.71)$ \\
\hline
\end{tabular}


Table 2 Comparisson for all questions on the MFSQ (median and range) before and six weeks after completed treatment using Wilcoxon signed rank test (paired)

\begin{tabular}{|c|c|c|c|}
\hline \multirow[t]{2}{*}{ Question } & \multirow{2}{*}{$\begin{array}{l}\text { MFSQ (before treatment) } \\
\text { Median (range) }\end{array}$} & \multicolumn{2}{|l|}{ MFSQ (six months after treatment) } \\
\hline & & Median (range) & $p$-value \\
\hline $\begin{array}{l}\text { 1. Are you satisfied with the extent of your sexual life at } \\
\text { the present moment? }(1=\text { very dissatisfied, } 7=\text { very satisfied })\end{array}$ & $\begin{array}{l}2.0(1-6) \\
n=60\end{array}$ & $\begin{array}{l}5.0(1-7) \\
n=60\end{array}$ & $<0.01^{*}(z-6.71)$ \\
\hline $\begin{array}{l}\text { 2. Approximately how many times, during the last month, } \\
\text { have you thought about sex or had sexual fantasies? } \\
\text { ( } 1=\text { never, } 7=>10 \text { times per day) }\end{array}$ & $\begin{array}{l}3.0(1-6) \\
n=60\end{array}$ & $\begin{array}{l}4.0(1.5-7) \\
n=60\end{array}$ & $<0.01^{*}(z-4.50)$ \\
\hline $\begin{array}{l}\text { 3. Does sexual activity give you pleasure? ( } 1=\text { no pleasure at all, } \\
7=a \text { lot of pleasure) }\end{array}$ & $\begin{array}{l}4.0(1-7) \\
n=53\end{array}$ & $\begin{array}{l}6.0(3-7) \\
n=54\end{array}$ & $<0.01^{*}(z-4.60)$ \\
\hline $\begin{array}{l}\text { 4. How often do you feel sexually excited or stimulated during } \\
\text { sexual activity? ( } 1=\text { never, } 7=\text { every time })\end{array}$ & $\begin{array}{l}4.0(1-7) \\
n=53\end{array}$ & $\begin{array}{l}6.0(3-7) \\
n=53\end{array}$ & $<0.01^{*}(z-5.10)$ \\
\hline $\begin{array}{l}\text { 5. How often do you acheive orgasm during sexual activity? } \\
(1=\text { never, } 7=\text { every time) }\end{array}$ & $\begin{array}{l}4.0(1-7) \\
n=53\end{array}$ & $\begin{array}{l}5.0(1-7) \\
n=53\end{array}$ & $0.01^{*}(z-2.50)$ \\
\hline $\begin{array}{l}\text { 6. How often is vaginal dryness a problem during sexual activity? } \\
(1=\text { every time, } 7=\text { never })\end{array}$ & $\begin{array}{l}4.0(1-7) \\
n=54\end{array}$ & $\begin{array}{l}5.0(1-7) \\
n=53\end{array}$ & $<0.01^{*}(z-3.13)$ \\
\hline 7. How often is sexual intercourse painful? ( 1 = every time, $7=$ never) & $\begin{array}{l}1.0(1-7) \\
n=53\end{array}$ & $\begin{array}{l}6.0(1-7) \\
n=53\end{array}$ & $<0.01^{*}(z-5.90)$ \\
\hline $\begin{array}{l}\text { 8. Are you satisfied with your partner as a lover? } \\
(1=\text { very dissatisfied, } 7=\text { very satisfied })\end{array}$ & $\begin{array}{l}7.0(2-7) \\
n=55\end{array}$ & $\begin{array}{l}6.0(2-7) \\
n=51\end{array}$ & $0.61(z-0.61)$ \\
\hline $\begin{array}{l}\text { 9. Are you satisfied with your partner as a fellow human } \\
\text { and friend? ( } 1=\text { very dissatisfied, } 7=\text { very satisfied })\end{array}$ & $\begin{array}{l}7.0(2-7) \\
n=54\end{array}$ & $\begin{array}{l}7.0(1-7) \\
n=53\end{array}$ & $0.10(z-1.71)$ \\
\hline $\begin{array}{l}\text { 10. How often have you had sexual intercourse during the } \\
\text { last month? }(1=\text { never, } 6=>8 \text { times })\end{array}$ & $\begin{array}{l}2.0(1-6) \\
n=60\end{array}$ & $\begin{array}{l}3.0(1-6) \\
n=59\end{array}$ & $<0.01^{*}(z-3.10)$ \\
\hline $\begin{array}{l}\text { 11. How often have you satisfied yourself, alone, during the } \\
\text { last month? ( } 1=\text { never, } 6=>8 \text { times) }\end{array}$ & $\begin{array}{l}1.5(1-6) \\
n=60\end{array}$ & $\begin{array}{l}3.0(1-6) \\
n=59\end{array}$ & $<0.01^{*}(z-4.10)$ \\
\hline $\begin{array}{l}\text { 12. How often do you avoid intercourse in order to avoid } \\
\text { pain? ( } 1 \text { = always, } 5=\text { never })\end{array}$ & $\begin{array}{l}2.0(1-5) \\
n=58\end{array}$ & $\begin{array}{l}4.0(1-5) \\
n=52\end{array}$ & $<0.01^{*}(z-5.80)$ \\
\hline
\end{tabular}

*Statistically significant increase in scores 